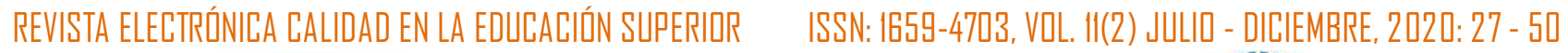

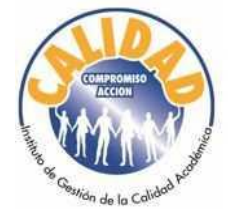

Vicerrectoría Académica

Instituto de Gestión de la Calidad Académica

Co-creando Excelencia

http://revistas.uned.ac.cr./index.php/revistacalidad

Согге electrónić: revistacalidad国uned.ас.сг

\title{
Análisis de las propiedades psicométricas del inventario Reflexiones sobre el estudio universitario
}

Analysis of the psychometric properties of the reflections on study inventory

\author{
Rocío Giselle Fernández-Da Lama1 \\ rfernandezdalama@uca.edu.ar \\ Pontificia Universidad Católica Argentina, Argentina \\ https://orcid.org/0000-0003-1529-2926
}

DOl: $h t t p: / / d x . d o i . o r g / 10.22458 /$ caes.v11i2.3318

Volumen 11, Número 2

30 de noviembre de 2020

pp. $27-50$

Recibido: 21 de enero del 2020

Aprobado: 28 de octubre del 2020

\footnotetext{
${ }^{1}$ Licenciada en Psicología en la Facultad de Psicología de la Universidad de Buenos Aires (2018), cursa el Profesorado de Educación Media y Superior en Psicología en la misma universidad. Actualmente trabaja en el Centro de Investigación de Psicología y Psicopedagogía de la Pontificia Universidad Católica Argentina. Las líneas de trabajo son educación superior, psicometría, y evaluación psicológica.
}

Análisis de las propiedades psicamétricas del inventario Reflexiones sobre el estudio universitario

Racía Giselle Fernández-Da Lama

DDI: http://dx.dai.arg//0.22458/caes.vlli2.3318

(c) $(1)(8)$

Artícula protegida par licencia Creative Cammans 


\section{Resumen}

El presente trabajo evalúa las propiedades psicométricas del inventario Reflexiones sobre el estudio universitario, el cual consta de 32 ítems. Este inventario se encuentra diseñado para medir el nivel de conciencia metacognitiva sobre los procesos de estudio del estudiantado. El diseño de este trabajo es no experimental, instrumental, y de corte transversal, con una muestra de 197 estudiantes de Psicología de la Universidad de Buenos Aires (80\% mujeres y $20 \%$ varones), de entre 18 y 48 años $(M=23.82, D E=6.14)$. El análisis de confiabilidad arroja un valor adecuado $(\alpha=.83)$ de consistencia interna. En cuanto a la validez de constructo, el Análisis Factorial Exploratorio muestra una estructura de 8 factores que explican el $67,72 \%$ de la varianza. Se discuten las bondades del instrumento a la luz de los resultados hallados y los aspectos teórico-conceptuales vigentes.

Palabras clave: metacognición, psicología, confiabilidad, validez.

\section{Abstract}

This work evaluates the psychometric properties of the Reflections on university study inventory, which consists of 32 items. This inventory is designed to measure the level of metacognitive awareness of the students' study processes. The design of this work is nonexperimental, instrumental, and cross-sectional, with a sample of 197 psychology students from the University of Buenos Aires ( $80 \%$ women and $20 \%$ men), between 18 and 48 years of age $(M=23.82, S D=6.14)$. The reliability analysis yields an adequate value $(\alpha=.83)$ of internal consistency. Regarding the construct validity, an Exploratory Factor Analysis shows a structure of 8 factors that explains $67.72 \%$ of the variance. The benefits of the instrument are discussed in light of the results found and the current theoretical-conceptual aspects.

Keywords: metacognition, psychology, reliability, validity.

\section{Introducción}

La metacognición es, en gran medida, referida al conocimiento sobre los procesos cognitivos - por ejemplo una persona puede conocer cuál es la capacidad de memoria, saber qué tipos de tareas le resultarán más difíciles que otras-, y a la regulación de dichos procesos - releer un texto la cantidad de veces necesarias para recordar la información, planificar estrategias a la hora de estudiar, entre otras acciones- (Flavell, 1971; Martí, 1995).

Análisis de las propiedades psicométricas del inventario Reflexiones sobre el estudio universitario

Racín Ciselle Fernández-Da Lama

DII: http://dx.dai.org//0.22458/caes.vlliz.3318

$$
\text { (c) } \underset{\mathrm{BY}}{\mathrm{N}}(\mathrm{NC}
$$

Artícula prategida par licencia Creative Cammans 
Desde los alcances actuales de la literatura científica, la metacognición se encuentra clasificada en tres aspectos principales.

El primer aspecto refiere a un conocimiento de carácter declarativo, el "saber qué...", en tanto la persona requiere de información para hacer frente a una situación determinada, donde existe una meta u objetivo cognitivo, por lo que el conocimiento sobre procesos de orden cognitivo como la memoria, la lectura, la escritura, entre otros, será esencial (Brown, 1987; Schraw y Moshman, 1995).

Por su parte, el segundo aspecto, de carácter procedimental, se halla referido al "saber cómo..." y permite lograr efectivamente la concreción de dichos objetivos cognitivos a través del conocimiento sobre cómo ejecutar determinadas acciones y procesos (Schraw y Moshman, 1995). Asimismo, este componente regulatorio desarrolla funciones como las de planificación, control, monitoreo, y evaluación del desempeño de los procesos cognitivos (Brown, 1987).

Posturas de autores más recientes (Mateos, 2001) corroboran la existencia de un tercer componente metacognitivo, el cual se denomina conocimiento de carácter condicional, acerca de qué estrategias utilizar ante determinadas situaciones, cuándo y dónde emplearlas. Esta postura se encuentra en consonancia con trabajos anteriores de Schraw y Moshman (1995), quienes indagaron con anterioridad sobre un tercer tipo de conocimiento metacognitivo sobre "por qué..." y "cuándo..." hacer uso de determinados procesos cognitivos.

\footnotetext{
Análisis de las propiedades psicométricas del inventario Reflexiones sobre el estudio universitario

Racín Giselle Fernández-Da Lama

DDI: http://dx.dai.org//0.22458/caes.v1li2.3318

(c) (1) (5)

Artícula prategida par licencia Creative Cammans
} 
Debido a la estrecha relación entre metacognición, conocimiento y aprendizaje, es que su estudio ha contribuido a la comprensión de los procesos involucrados en la resolución de problemas en diferentes áreas de conocimientos (Águila, 2014; Osses-Bustingorry y Jaramillo-Mora, 2008; Sandia-Rondel, 2004). El creciente interés por la metacognición en el ámbito académico y científico puede atribuirse a distintos factores. Un factor sería el desarrollo de la psicología cognitiva, y su relación con la educación, lo cual introdujo en gran medida el planteamiento del estudiante en tanto sujeto activo que procesara información de manera relativamente independiente del trabajo del profesor o de la influencia del medio externo, en lugar de tratarlo como un mero sujeto respondiente a estímulos externos e internos (Mayer, 1992). Esta visión, en complementariedad con los enfoques constructivistas, los cuales resaltan el aprendizaje como una instancia de construcción a partir de conocimientos y experiencias diversas por vía de la enseñanza (Coll, 1996), arroja luz sobre ciertos aspectos que habían quedado rezagados en las investigaciones de corte conductista, especialmente referidos a procesos como el aprendizaje (Aparicio y RodríguezMoneo, 2010).

Otro factor de importancia ha sido el crecimiento y desarrollo de la idea del "conocimiento" como un bien social y esencial para promover cambios tendientes al bienestar y el progreso de las naciones por medio de educación de calidad (Unesco, 1998), especialmente en la educación superior. En las últimas décadas se han dado cambios en lo metodológico y lo epistemológico en la enseñanza actual, bregando por formas más flexibles, amplias y humanistas que las tradicionales para la construcción de conocimientos significativos (Torres-Salas, 2010).

Los factores anteriormente mencionados contribuyen al crecimiento del interés a nivel investigativo y académico (Glaser, 1994) de la metacognición y su desarrollo, especialmente

Análisis de las propiedades psicométricas del inventario Reflexiones sobre el estudio universitario

Racín Giselle Fernández-Da Lama

DII: http://dx.dai.org//0.22458/caes.v1li2.3318

(c) (i) \$

Artícula prategida por licencia Ereative Cammans 
en el campo educativo. En gran medida, los estudiantes deben enfrentarse a nuevas tareas de aprendizaje, con lo cual se convierte en una necesidad que estos puedan determinar sus potencialidades y debilidades en cuanto al conocimiento de sus propios procesos cognitivos, reflexionar sobre los conocimientos aprendidos y aplicarlos a situaciones novedosas, así como regular dichos procesos en vía de su optimización (Gutiérrez-Rico, 2005; Nickerson, 1984; Osses-Bustingorry y Jaramillo-Mora, 2008). Esta necesidad de "aprender a aprender" se acompaña de adecuadas metodologías de enseñanza y aprendizaje centradas en el "enseñar a aprender" (Osses-Bustingorry y Jaramillo-Mora, 2008), no solo pueden mejorarse los procesos de aprendizaje del estudiantado, sino además gestarse en este la capacidad de aprender independientemente (Chadwick, 1985; Pozo, 1990).

En consonancia con lo anterior, numerosos trabajos aportan evidencias acerca de su relación con la mejoría en el rendimiento académico en estudiantes de distintos niveles (Ochoa-Angrino, Aragón-Espinosa, Correa-Restrepo, y Mosquera, 2008; Sandia-Rondel, 2004), especialmente el nivel medio (e.g. Altamirano-Laura, 2015; Llanos-Luyo, 2018; Maturano, Mazzitelli, y Ascensión, 2009) y universitario (e.g. Escanero-Marcén, Soria, Escanero-Ereza, y Guerra-Sánchez, 2013; Vrugt y Oort, 2008; Young y Fry, 2008); otras investigaciones destacan el papel de la dimensión metacognitiva con el fin de lograr estudiantes que autorregulen su propio aprendizaje (Doganay y Demir, 2011; Klimenko y Alvares, 2009) y desarrollen un pensamiento crítico (Botero-Carvajal, Alarcón, PalominoAngarita, y Jiménez-Urrego, 2017; Magno, 2010).

La evaluación de la metacognición es harto compleja debido a que este constructo, si bien no es un proceso meramente intrínseco, no se presenta como un comportamiento explícito y fácilmente observable (Jaramillo y Osses, 2012). En este sentido, si bien una técnica de

Análisis de las propiedades psicométricas del inventario Reflexiones sobre el estudio universitario

Racín Giselle Fernández-Da Lama

DII: http://dx.dai.org//0.22458/caes.vlliz.3318

(c) (i) \$

Artícula prategida por licencia Ereative Cammans 
evaluación psicológica presenta cierta capacidad discriminatoria de la variable que desea medir, siempre se hacen presentes limitaciones en las mediciones que se efectúan, sean estas por sesgos socioculturales, falencias del basamento teórico de la prueba, entre otros (Meneses et al., 2014). Por consiguiente, el poder evaluar aspectos y procesos cognitivos que resultan ser complejos, tales como la metacognición, implica analizar únicamente aquellas conductas que se asocian y son dirigidas por los mismos procesos de carácter cognitivo que se pretenden estudiar (Jiménez-Rodríguez, 2004).

Distintos instrumentos para su evaluación pueden clasificarse en dos grandes categorías. Por un lado, los instrumentos basados en el reporte que hace el sujeto de su propia actividad, como cuestionarios y entrevistas; y por otro lado, medidas objetivas del comportamiento, reportadas por medio de observaciones sistemáticas y de protocolos de pensamiento en voz alta (Ozturk, 2017). La primera categoría presenta mayor amplitud en su desarrollo y deriva en una gran cantidad de instrumentos.

Dentro de los distintos instrumentos de autorreporte actualmente vigentes que evalúan la metacognición pueden nombrarse el Inventario de Estrategias Metacognitivas (O'Neil y Abedi, 1996), el Inventario del Conocimiento Metacognitivo de las Estrategias de Interpretación de Lectura (MARSI, Mokhtari \& Reichard, 2002), y el Inventario de Habilidades Metacognitivas (MAI, Schraw \& Dennison, 1994).

Un instrumento de reciente desarrollo en la Argentina es el inventario Reflexiones sobre el estudio universitario (Palacios y Schinella, 2017), el cual ha registrado adecuadas propiedades psicométricas en su evaluación de nivel de conciencia metacognitiva sobre los procesos de estudio en estudiantes de la carrera de Medicina de la Universidad Nacional de La Plata. Palacios y Schinella tomaron como base para la elaboración del inventario los

Análisis de las propiedades psicométricas del inventario Reflexiones sobre el estudio universitario

Racín Giselle Fernández-Da Lama

DUI: http://dx.dai.org//0.22458/caes.vlliz.3318

(c) (i) \$

Artícula prategida por licencia Lreative Cammans 
instrumentos MAI (Schraw \& Dennison, 1994) y la Lista Control de Hábitos de Estudio de Agudo y Moraschi (Agudo \& Moraschi, 1999).

El objetivo del presente trabajo es evaluar las propiedades psicométricas del inventario Reflexiones sobre el estudio universitario (Palacios y Schinella, 2017) administrado a estudiantes de la carrera de Psicología de la Universidad de Buenos Aires. Con este fin, se analizan las propiedades métricas de los ítems que componen la escala, y se examina la estructura factorial de esta y su nivel de confiabilidad.

\section{Método}

\section{Tipo de estudio}

Se trata de un estudio de carácter instrumental por evaluar aspectos relacionados con la construcción y adaptación de instrumentos de medición (Montero y León, 2007).

\section{Muestra}

La muestra está compuesta por 197 estudiantes (80\% varones y $20 \%$ mujeres), de entre 18 y 48 años ( $M=23.82, D E=6.14)$, seleccionados mediante un muestreo intencional simple. Se incluyen en la investigación estudiantes de todos los años de la carrera -primer año $(13,7 \%)$; segundo año $(15,7 \%)$; tercer año $(28,4 \%)$, cuarto año $(18,8 \%)$; quinto año $(8,1 \%)$, y sexto año $(15,2 \%)$ - provenientes de la Facultad de Psicología de la Universidad de Buenos Aires.

\section{Procedimiento}

El alumnado participante del estudio fue contactado por correo electrónico, contó con el aval del grupo docente de las distintas comisiones evaluadas. A cada participante se le informó

Análisis de las propiedades psicométricas del inventario Reflexiones sobre el estudio universitario

Racín Giselle Fernández-Da Lama

DII: http://dx.dai.org//0.22458/caes.vlliz.3318

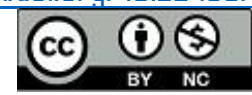

Artícula prategida par licencia Creative Cammans 
del carácter voluntario y anónimo de sus respuestas por medio de la entrega de un consentimiento informado, así como la posibilidad de dejar de responder este en cualquier momento de su aplicación.

\section{Instrumentos}

-Encuesta sociodemográfica, la cual contiene aspectos tales como sexo, edad, y año actual de cursada en la carrera.

-Inventario Reflexiones sobre el estudio universitario (Palacios y Schinella, 2017), diseñado por investigadores de la Universidad Nacional de la Plata para ser administrado a estudiantes universitarios vía online, con evidencias adecuadas de validez de constructo y de contenido, así como una consistencia interna apropiada para cada una de sus dimensiones. Este instrumento evalúa por medio 32 ítems, con formato de respuesta Likert de cinco posiciones, (5=Completamente de acuerdo; $1=$ Completamente en desacuerdo) el conocimiento que el estudiante tiene sobre la percepción de sí mismo en el estudio (total de 10 ítems), las acciones específicas sobre el estudio (total de 12 ítems), y el autoseguimiento en el estudio (total de 10 ítems). Asimismo, se le da la posibilidad al estudiante de no responder si no está seguro de su respuesta, puntuando dicha opción como cero. La puntuación de los resultados se realiza mediante la sumatoria de los puntajes parciales arrojados por los tres tipos de conocimiento ya mencionados, obteniéndose un puntaje total, que varía de un puntaje mínimo de 0 a un puntaje máximo de 160, indicador de la metacognición sobre el estudio del evaluado. Además, el inventario cuenta con tres preguntas de respuesta abierta donde el estudiante responde sin ningún tipo de restricción, y un espacio para dejar sus observaciones. Las tres preguntas en orden son: ¿Qué autocrítica tienes como estudiante?: ¿Qué tipo de lecturas/temas/materias de la carrera te

Análisis de las propiedades psicométricas del inventario Reflexiones sobre el estudio universitario

Racín Giselle Fernández-Da Lama

DII: http://dx.dai.org//0.22458/caes.vlliz.3318

(c) (i) \$)

Artícula prategida par licencia Ereative Cammans 
$\overline{\text { resultan más difíciles para estudiar?: ¿Qué acciones/estrategias te resultan más exitosas en }}$ el estudio de las materias universitarias?

\section{Análisis de los datos}

El análisis métrico de los ítems del instrumento se efectúa mediante el cálculo de medias y desviaciones estándar. Para determinar la confiabilidad del instrumento se calcula el valor Alfa de Cronbach, el cual determina el nivel de consistencia interna de la escala. En cuanto a la estructura factorial de la escala, se desarrolla un análisis factorial exploratorio con método de Análisis de Componentes Principales y rotación Varimax. Los análisis se llevan a cabo con el programa IBM SPSS versión 21.

\section{Resultados}

Análisis de los ítems

En la Tabla 1 pueden apreciarse los valores medios y desvíos de todos los ítems. Los indicadores que presentan valores medios de acuerdo con los más altos son el ítem 21 ("retrocedo y vuelvo a leer cuando la información no me resulta clara"), el ítem 23 ("trato de usar estrategias de estudio que en el pasado han sido exitosas"), y el ítem 8 ("me doy cuenta si he comprendido bien"). En contraposición, el ítem con menor valor medio de acuerdo son el 17 ("hago diagramas, cuadros, gráficos, dibujos para reducir la información"). En síntesis, la mayoría de los ítems se posicionan en un valor medio entre de acuerdo y desacuerdo, lo cual indica el predominio de cierta incertidumbre e indecisión por parte de los estudiantes.

\section{Análisis de la confiabilidad}

El nivel de confiabilidad del instrumento es estimado mediante el cálculo del Coeficiente Alfa de Cronbach, el cual es de .83. Esto arroja una consistencia interna adecuada de la escala

\footnotetext{
Análisis de las propiedades psicamétricas del inventario Reflexiones sobre el estudia universitario

Racín Giselle Fernández-Da Lama

DDI: http://dx.dai.arg/l0.22458/caes.vlli2.3318

(c)

Artícula prategida por licencia Ereative Cammans
} 
en general (Cronbach \& Shavelson, 2004). Para cada subescala, el alfa de Cronbach es de $\alpha=.68$ para Percepción sobre sí mismo; de $\alpha=.68$ para Acciones específicas frente al estudio con; y en último lugar, de $\alpha=.59$ para Autoseguimiento en el estudio. En cuanto a las correlaciones ítem-test corregidas, todas se ubican en el rango de .20 a .54, exceptuando los ítems 17, 26 y 27 (Tabla 1). 


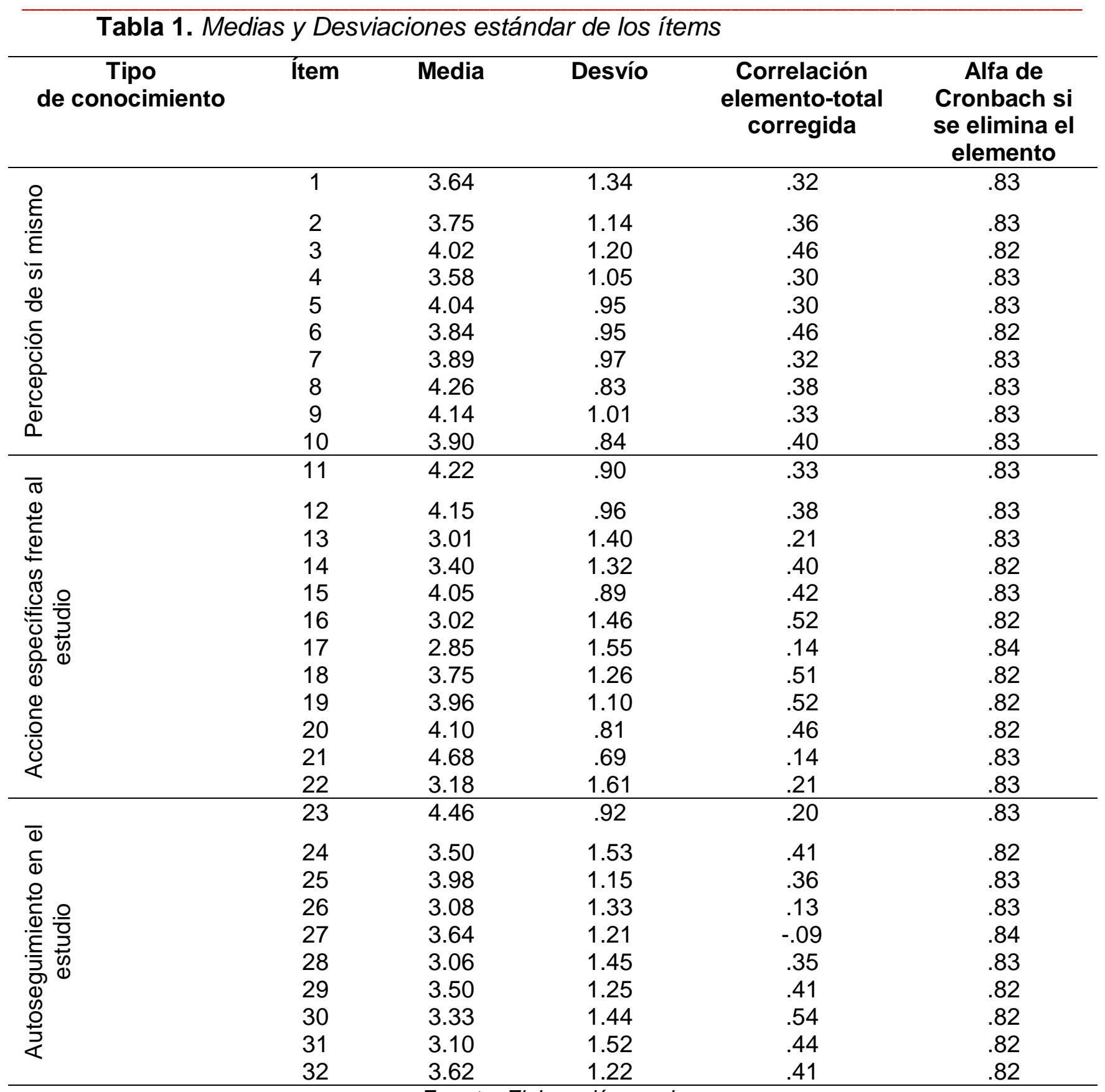

Fuente: Elaboración propia 


\section{Análisis factorial}

Previo a la elaboración del análisis factorial exploratorio, se analiza si la matriz de datos es apropiada para ejecutar un análisis factorial. La prueba de esfericidad de Bartlett $\left(X^{2}{ }_{(406)}=\right.$ $2799.43, p<.001)$ indica que los ítems no provienen de una matriz identidad. El coeficiente de Kaiser-Meyer-Olkin arroja un valor de .661, lo cual indica que es apropiado aplicar el análisis factorial. Se desarrolla un primer análisis factorial exploratorio, en el cual se halla que el $69,09 \%$ de la varianza total acumulada es explicada por 10 factores. Sin embargo, en la matriz rotada, los ítems 17, 26, y 27 del inventario presentaron cargas factoriales al criterio mínimo de inclusión (.30) en todos los factores.

Se efectúa un segundo análisis factorial exploratorio excluyendo los ítems 17, 26, y 27. Nuevamente los datos son propicios para la elaboración de dicho procedimiento: prueba de esfericidad $\left(X^{2}{ }_{(406)}=2336.86, p<.001 ; \mathrm{KMO}=.685\right)$, hallándose 9 factores que explican un $67,72 \%$ de la varianza total acumulada. Sin embargo, teniendo en cuenta las cargas factoriales, solo los factores I, II, III, IV, V, VI, VIII, y IX presentaron un valor explicativo de la estructura factorial de la escala (explicando el $67,72 \%$ de la varianza), ya que el factor restante se encuentra constituido únicamente por dos indicadores. La matriz de factores resultantes tras la rotación es expuesta en la Tabla 2, donde se presentan las saturaciones por encima de .30 de los indicadores correspondientes.

El factor I explica el $21,41 \%$ de la varianza y satura fuertemente en los ítems 4,5 y 7 , los cuales corresponden a Percepción de sí mismo - conocer el propio desempeño tras un examen, detección de la información más relevante, y facilidad para recordar información-.

El factor II explica el $8,35 \%$ de la varianza y satura en los ítems 1,2, y 15, los cuales remiten tanto a Percepción sobre sí mismo — propuesta de metas específicas al estudiar,

Análisis de las propiedades psicométricas del inventario Reflexiones sobre el estudio universitario

Racín Giselle Fernández-Da Lama

DII: http://dx.dai.org//0.22458/caes.vlliz.3318

(c) (i) \$

Artícula prategida por licencia Lreative Cammans 
distribución del tiempo para un mejor aprovechamiento en el estudio-, y a Acciones específicas en el estudio —centrarse en la información más relevante-.

El factor III explica el $8,14 \%$ de la varianza, este satura en los ítems 12, 19, 20, y 21, pertenecientes a Acciones específicas en el estudio — detenerse y reflexionar al encontrarse con información relevante, relacionar lo nuevo con conocimientos previos sobre la temática, revisar las ideas ante la confusión, y releer al no resultar clara la información-.

El factor IV explica el 6,35\% de la varianza y satura en los ítems 14, 16, 31 y 32, los cuales pertenecen a Acciones específicas en el estudio - solicitar ayuda al no comprender, proponerse ejemplos para favorecer la comprensión-, y Autoseguimiento en el estudio autoformularse preguntas para controlar el propio aprendizaje, y revisar la comprensión de relaciones de importancia-.

El factor V explica el 5,93 \% de la varianza, satura en los ítems 3, 10, y 28, pertenecientes a Percepción sobre sí mismo - conocimiento de puntos débiles y fuertes en el estudio, saberse capaz de poder resolver los problemas que se presenten a la hora de estudiar-, y Autoseguimiento en el estudio - poder determinar si la estrategia de estudio empleada es errónea-.

El factor VI explica el 5,34\% de la varianza y satura en los ítems 6,13 , y 18, los cuales refieren a la Percepción sobre sí mismo — saberse eficiente para resumir y sintetizar la información-, y las Acciones en el estudio — sintetizar lo aprendido luego de estudiar, y tratar de reformular nociones con las propias palabras-.

Análisis de las propiedades psicométricas del inventario Reflexiones sobre el estudio universitario

Racín Giselle Fernández-Da Lama

DII: http://dx.dai.org//0.22458/caes.vlliz.3318

(c) $\underset{\mathrm{BY}}{\mathrm{NC}}$

Artícula prategida par licencia Creative Cammans 
El factor VIII explica el 3,77\% de la varianza, satura en los ítems 8,22 , y 23, los cuales refieren a la Percepción sobre sí mismo — darse cuenta de la propia comprensión, a las Acciones específicas en el estudio - organizar el estudio en etapas-, y Autoseguimiento en el estudio —emplear estrategias de estudio que han sido exitosas en el pasado-.

El factor IX explica el 3,64\% de la varianza, saturando en los ítems 9, 24, y 25, los cuales refieren a la Percepción sobre sí mismo — consciencia de los propios hábitos de estudio-, y a Autoseguimiento en el estudio -emplear estrategias de estudio con un motivo específico, y adecuar la forma de estudiar dependiendo de las situaciones-. 
Tabla 2. Factores resultantes de la rotación y los indicadores con el peso factorial correspondiente en el segundo análisis factorial exploratorio.

\begin{tabular}{|c|c|c|c|c|c|c|c|c|c|}
\hline \multirow[t]{2}{*}{ Ítems } & \multicolumn{9}{|c|}{ Factores } \\
\hline & I & II & III & IV & V & VI & VII & VIII & IX \\
\hline 4 & .49 & & & & & & & & \\
\hline 5 & .70 & & & & & & & & \\
\hline 7 & .76 & & & & & & & & \\
\hline 1 & & .77 & & & & & & & \\
\hline 2 & & .69 & & & & & & & \\
\hline 15 & & .58 & & & & & & & \\
\hline 12 & & & .73 & & & & & & \\
\hline 19 & & & .55 & & & & & & \\
\hline 20 & & & .68 & & & & & & \\
\hline 21 & & & .76 & & & & & & \\
\hline 14 & & & & .80 & & & & & \\
\hline 16 & & & & .56 & & & & & \\
\hline 31 & & & & .55 & & & & & \\
\hline 32 & & & & .42 & & & & & \\
\hline 3 & & & & & .74 & & & & \\
\hline 10 & & & & & .45 & & & & \\
\hline 28 & & & & & .69 & & & & \\
\hline 6 & & & & & & .74 & & & \\
\hline 13 & & & & & & .58 & & & \\
\hline 18 & & & & & & .60 & & & \\
\hline 29 & & & & & & & .83 & & \\
\hline 30 & & & & & & & .52 & & \\
\hline 8 & & & & & & & & .38 & \\
\hline 22 & & & & & & & & .72 & \\
\hline 23 & & & & & & & & .71 & \\
\hline 9 & & & & & & & & & .77 \\
\hline 24 & & & & & & & & & .50 \\
\hline 25 & & & & & & & & & .48 \\
\hline
\end{tabular}

Fuente: Elaboración propia

Análisis de las propiedades psicométricas del inventario Reflexiones sobre el estudio universitario

Racía Giselle Fernández-Da Lama

DIII: http://dx.dai.org/10.22458/caes.vlliz.3318

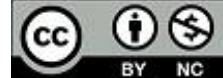

Artícula prategida par licencia Creative Cammans 
Con respecto al análisis de las preguntas de respuesta abierta formuladas al final del instrumento, se procesa el contenido de estas mediante la herramienta informática denominada "nube de palabas", con el fin de identificar los términos más repetidos por los estudiantes (ver Figura 1).

En cuanto a los aspectos que el estudiantado menciona como autocrítica en mayor medida, se hallaron referidos a las dificultades para organizarse en cuanto al estudio, el llevar la lectura de textos al día a pesar de otras responsabilidades, el dejar gran cantidad de textos para leer poco tiempo antes de los exámenes.

Con respecto al tipo de lecturas/temas/materias que les resultan más difíciles para aprender, los estudiantes hacen hincapié en las dificultades de aprender conceptos de memoria. Asimismo, establecieron que tanto las asignaturas con contenidos de neurofisiología, estadística, matemáticas, biología, e investigación, como aquellas que contienen una elevada cantidad de contenido psicoanalítico, les reportan grandes dificultades en su aprendizaje.

Acerca de las acciones/estrategias exitosas al momento de estudiar, surgen, en gran medida la confección y el uso de resúmenes, mantener la lectura de los textos al día para cada clase, resaltando la información más relevante, el aprender información de memoria, y el trabajar con otros compañeros en grupo.

Análisis de las propiedades psicométricas del inventario Reflexiones sobre el estudio universitario

Racín Giselle Fernández-Da Lama

DII: http://dx.dai.org//0.22458/caes.v1li2.3318

(c) (1) (8)

Artícula prategida par licencia Creative Cammans 


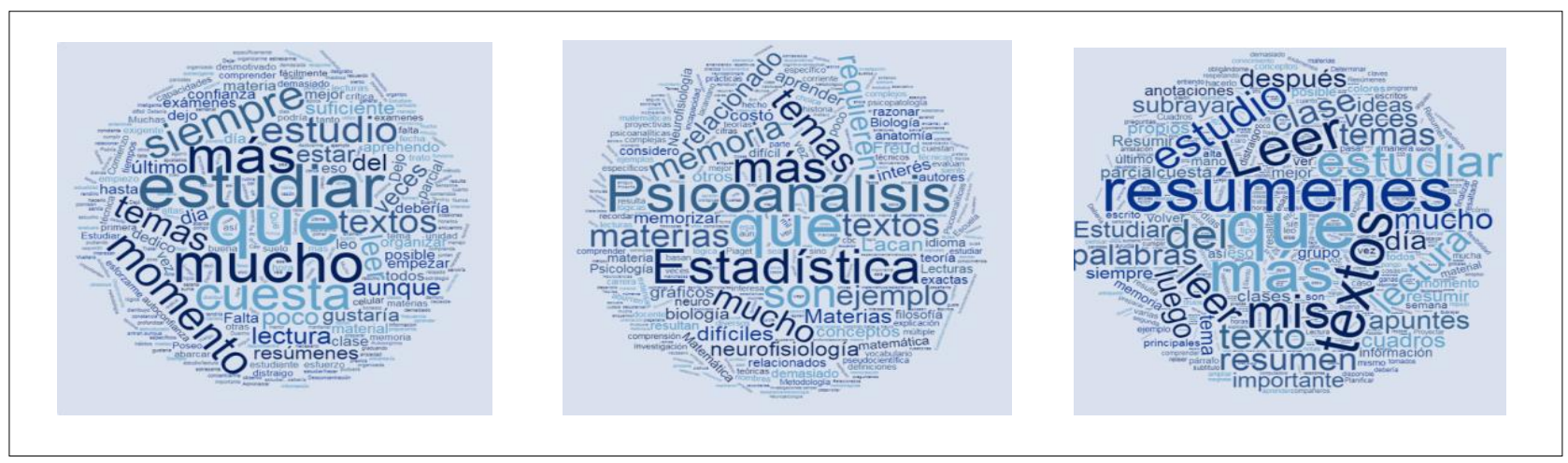

Figura 1. Nube de palabras, preguntas uno a tres ordenadas de izquierda a derecha. Pregunta 1: ¿Qué autocrítica tienes como estudiante?; pregunta 2: ¿Qué tipo de lecturas/temas/materias de la carrera te resultan más difíciles para estudiar?; pregunta 3: ¿Qué acciones/estrategias te resultan más exitosas en el estudio de las materias universitarias?

\section{Discusión}

El presente artículo se propone desarrollar un análisis de las propiedades psicométricas del inventario Reflexiones sobre el estudio universitario (Palacios y Schinella, 2017) en una muestra de estudiantes de la carrera de Psicología de la Universidad de Buenos Aires. Dicho inventario, de reciente desarrollo, presenta adecuadas características psicométricas en la evaluación de las características estudiantiles metacognitivas en una muestra de estudiantes de la carrera de Medicina, por lo que se consideró de relevancia el comprobar las características técnicas del instrumento en su evaluación de estudiantes de otra carrera relacionada a la salud, a saber, Psicología.

Análisis de las propiedades psicométricas del inventario Reflexiones sobre el estudio universitario

Racín Ciselle Fernández-Da Lama

DII: http://dx.dai.org//0.22458/caes.vlliz.3318

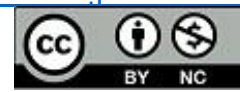

Artícula prategida par licencia Creative Cammans 
Los análisis descriptos de los ítems arrojan puntajes medios más elevados de los ítems provenientes del tipo de conocimiento metacognitivo referido a las Acciones específicas frente al estudio, lo cual indica un mayor acuerdo por parte de los estudiantes en el grado de consciencia que manejan sobre las estrategias, tales como pensar en todo lo que necesitan antes de comenzar a estudiar, detenerse y reflexionar cuando encuentran información importante, centrar la atención intencionalmente en información importante, revisar sus ideas cuando siente confusión, y retroceder y volver a leer cuando la información no resulta clara. Sin embargo, el ítem con valor medio más bajo dentro de este tipo de conocimiento es el 17 , el cual apunta al uso de herramientas visuales, tales como diagramas, cuadros, gráficos, etc., para reducir la información a estudiar. Esta información se complementa con lo respondido por el estudiantado en las preguntas de respuesta abierta, donde indicaron un mayor uso de resúmenes y el aprender información de memoria.

Un dato de marcada importancia es el predominio de incertidumbre por parte de los estudiantes en los tres tipos de conocimiento metacognitivo al reaccionar antes las afirmaciones. Esto se evidencia al comprobar que la mayoría de las respuestas se ubicó en la opción 3 ("ni de acuerdo ni en desacuerdo"). Complementando esto con las opiniones reportadas por el alumnado, permiten localizar ciertas dificultades en este acerca de la variedad de estrategias de estudio que pueden implementarse y que el estudiantado parece desconocer.

En pos de analizar los valores de confiabilidad, se calcula el Alfa de Cronbach de la totalidad de la escala $(\alpha=.83$ ), y las correlaciones ítem-test (rango entre .20 a .54), obteniéndose adecuados valores de la consistencia interna del inventario. Asimismo, la

Análisis de las propiedades psicométricas del inventario Reflexiones sobre el estudio universitario

Racín Giselle Fernández-Da Lama

DII: http://dx.dai.org//0.22458/caes.vlliz.3318

(c) (i) (5)

Artícula prategida por licencia Lreative Cammans 
estimación de la consistencia interna de las subescalas del instrumento, arroja valores entre moderados y cuestionables (Cronbach \& Shavelson, 2004) para estas.

En cuanto a las evidencias de validez de constructo del instrumento, el análisis factorial exploratorio por medio de Análisis de componentes principales y rotación Varimax desarrollado, extrajo nueve factores en total que explicaron un $67,72 \%$ de la varianza total. Sin embargo, de los nueve factores extraídos solo pueden considerarse aquellos que cuenten con al menos tres indicadores por factor, resultando así en un total de ocho factores que representan la estructura dimensional del constructo.

Al comparar los resultados obtenidos en este estudio con los reportados por Palacios y Schinella (2017), pueden hallarse diferencias importantes en la estructura factorial. En primer lugar, los autores aíslan 11 factores, de los cuales, solo seis de ellos presentan como mínimo tres indicadores por factor. De esta manera, resulta en gran medida diferente en la cantidad de factores la solución factorial de ambas investigaciones. Además, un análisis minucioso revela que los factores resultantes no se encuentran compuestos de la misma manera. Este hallazgo revela discrepancias que pueden deberse a diferencias en las poblaciones estudiadas, principalmente en lo concerniente al tipo de estudio, contenidos, y tareas al que son sometidos los estudiantes.

Se plantea como necesario el análisis de muestras de mayor tamaño, así como pertenecientes a estudiantes universitarios de distintas carreras, con el fin de corroborar si la estructura factorial hallada en el presente trabajo se replica. En vías de aportar mayores evidencias de validez de constructo sobre la escala sería recomendable la elaboración de un análisis factorial confirmatorio. Además, para aportar evidencias para otro tipo de validez,

Análisis de las propiedades psicométricas del inventario Reflexiones sobre el estudio universitario

Racín Giselle Fernández-Da Lama

DII: http://dx.dai.org//0.22458/caes.vlliz.3318

(c) (1) $\$$

Artícula prategida par licencia Creative Cammans 
tales como de criterio, se plantea de utilidad el evaluar la escala en conjunto con otros instrumentos que valoren la metacognición.

\section{Referencias}

Agudo, M. C. \& Moraschi, M. (1999). Reflexiones sobre el oficio de estudiar. Un portafolio para el estudio independiente. Buenos Aires: Editorial de la Universidad Nacional de La Plata.

Águila, E. (2014). Habilidades y estrategias para el desarrollo del pensamiento crítico y creativo en alumnado de la Universidad de Sonora (tesis Doctoral). Universidad de Extremadura, Badajoz, España.

Altamirano, L. (2015). Aplicación de instrumentos de evaluación que permite el desarrollo de la metacognición en los estudiantes del tercer grado "b" en el área de formación ciudadana y cívica de la institución educativa secundaria José María Arguedas Altamirano del distrito Kaquiabamba de la provincia de Andahuaylas (tesis de grado). Universidad Nacional de San Agustín de Arequipa, Arequipa, Perú.

Aparicio, J. J. y Rodríguez, M. (2000). Los estudios sobre el cambio conceptual y las aportaciones de la Psicología del Aprendizaje. Tarbiya Revista de Investigación e Innovación Educativa, Monográfico Cambio Conceptual y Educación, 15(3), 13-30.

Botero-Carvajal, A., Alarcón, D., Palomino-Angarita, D. y Jiménez-Urrego, A. (2017). Pensamiento crítico, metacognición y aspectos motivacionales: una educación de calidad. Poiesis, 1(33), 85-103. doi: 10.21501/16920945.2499

Brown, A. L. (1987). Metacognition, executive control, self-regulation, and other more mysterious mechanisms. En F. Weinert, y R. Kluwe (Eds.). Metacognition, motivation and understanding (pp. 65-116). New Jersey, USA: LEA.

Chadwick, C. (1985). Estrategias Cognitivas, Metacognición y el uso de Microcomputadores en la Educación. Planiuc, 4(7).

Coll, C. (1996). Aprendizaje escolar y construcción del conocimiento. Barcelona, España: Paidós.

Análisis de las propiedades psicamétricas del inventario Reflexiones sobre el estudia universitario

Racín Ciselle Fernández-Da Lama

DII: http://dx.dai.org//0.22458/caes.vlli2.3318

(c) $(1)(9)$

Artícula protegida par licencia Creative Cammans 
Cronbach, L. \& Shavelson, R. (2004). My current thoughts on coefficient alpha and successor procedures. Educational and psychological measurement, 64(3), 391-418. Recuperado de http://citeseerx.ist.psu.edu/viewdoc/download?doi=10.1.1.575.7830\&rep=rep1\&type= pdf

Doganay, A. y Demir, O. (2011). Comparison of the Level of Using Metacognitive Strategies during Study between High Achieving and LowAchieving Prospective Teachers. Educational Sciences: Theory y Practice ,11(4), 2036-2043.

Escanero-Marcén, J., Soria, M., Escanero-Ereza, M. y Guerra-Sánchez, M. (2013). Influencia de los estilos de aprendizaje y la metacognición en el rendimiento académico de los estudiantes de fisiología. Fundación Educación Médica, 16(1), 2329.

Flavell, J. H. (1971). First discussant's comments: What is memory development the development of? Human Development, 14, (4), 272-278. doi: 10.1159/000271221

Glaser, R. (1994). Learning theory and instruction. En G. D’Ydewalle, P. Eelen, y B. Bertelson (eds.). International perspectives on psychological science. New Jersey, USA: Erlbaum.

Gutiérrez-Rico, D. (2005). Fundamentos teóricos para el estudio de las estrategias cognitivas y metacognitivas. Investigación Educativa Duranguense, 4, 21-28.

Jaramillo, S. y Osses, S. (2012). Validación de un Instrumento sobre Metacognición para Estudiantes de Segundo Ciclo de Educación General Básica. Estudios pedagógicos (Valdivia), 38(2), 117-131.

Jiménez-Rodríguez, V. (2004). Metacognición y comprensión de la lectura: evaluación de los componentes estratégicos (procesos y variables) mediante la elaboración de una escala de conciencia lectora (ESCOLA) (tesis Doctoral). Universidad Complutense de Madrid, Madrid, España. Recuperado de https://eprints.ucm.es/5337/1/T27494.pdf

Klimenko, O. y Alvares, J. (2009). Aprender cómo aprendo: la enseñanza de estrategias metacognitivas. Educación y Educadores, 12(2), 11-28.

Análisis de las propiedades psicamétricas del inventario Reflexiones sobre el estudia universitario

Racín Ciselle Fernández-Da Lama

DII: http://dx.dai.org//0.22458/caes.vlliz.3318

(c) $\underset{\mathrm{BY}}{\mathrm{B} \mathrm{NC}}$

Artícula prategida par licencia Creative Cammans 
Llanos-Luyo, M. (2018). Promoviendo en el trabajo colegiado el uso de estrategias metacognitivas incrementaremos el nivel de logro en la competencia lectora en los estudiantes del VI ciclo del nivel secundario de la IE. № 6008 José Antonio Dapelo (tesis de grado). Universidad Peruana Cayetano Heredia, Perú. Recuperado de http://repositorio.upch.edu.pe/handle/upch/2987

Magno, C. (2010). The role of metacognitive skills in developing critical thinking. Metacognition Learning, 5(2), 137-156. doi: 10.1007/s11409-010-9054-4

Martí, E. (1995). Metacognición: Entre la fascinación y el desencanto. Infancia y Aprendizaje, 72, 9-32.

Mateos, M. (2001). Metacognición y Educación. Buenos Aires, Argentina: AIQUE.

Mayer, R. E. (1992). A series of books in psychology. Thinking, problem solving, cognition. New York, USA: W H Freeman/Times Books/ Henry Holt y Co.

Meneses, J., Barrios, M., Bonillo, A., Cosculluela, A., Lozano, L. M., Turbany, J., \& Valero, S. (2014). Psicometría. Barcelona: Editorial UOC.

Maturano, C., Mazzitelli, C. y Macías, A. (2009). Detección de dificultades básicas de estudiantes de escuela secundaria en la comprensión de un texto de física. LatinAmerican Journal of Physics Education, 4(1), 160-167.

Mokhtari, K. y Reichard, C. (2002). Assessing Students' Metacognitive Awareness of reading Strategies. Journal of Educational Psychology, 94 (2), 249-259.

Montero, I. \& León, O. (2007). A guide for naming research studies in Psychology. International Journal of Clinical and Health Psychology, 7(3), 847-862. Recuperado de

http://webcache.googleusercontent.com/search?q=cache:http://www.psiencia.org/doc s/GNEIP07_es.pdf

Nickerson, R. (1984). Kinds of Thinking Taught in Currents Programs. Educational Leadership, 42(1), 26-36

O'Neil, H. F. y Abedi, J. (1996). Reliability and Validity of a State Metacognitive Inventory: Potential for Alternative Assessment. The Journal of Educational Research, 89(4), 234-245. doi:10.1080/00220671.1996.9941208

Análisis de las propiedades psicométricas del inventario Reflexiones sobre el estudio universitario

Racín Ciselle Fernández-Da Lama

DUl: http://dx.dai.arg//0.22458/caes.vlliz.3318

(c) $\underset{\mathrm{BY}}{\mathrm{B}} \mathrm{\textrm {NC }}$

Artícula prategida par licencia Creative Cammans 
Ochoa-Angrino, S., Aragón-Espinosa, L., Correa-Restrepo, M. y Mosquera, S. (2008). Funcionamiento metacognitivo de niños escolares en la escritura de un texto narrativo antes y después de una pauta de corrección conjunta. Acta Colombiana de Psicología, 11(2), 77-88.

Osses-Bustingorry, S. y Jaramillo, M. (2008). Metacognición: un camino para aprender a aprender. Estudios Pedagógicos, 34(1), 187-197.

Ozturk, N. (2017). Assessing Metacognition: Theory and Practices. International Journal of Assessment Tools in Education, 4(2), 134-148.

Palacios, A. \& Schinella, G. (2017). Diseño y validación de un instrumento para evaluar la metacognición sobre el estudio en estudiantes de medicina. Revista de Educación de la Universidad de Granada, 24, 9-28. Recuperado de https://www.researchgate.net/publication/319065547_Diseno_y_validacion_de_un_in strumento_para_evaluar_la_metacognicion_sobre_el_estudio_en_estudiantes_de_m edicina

Pozo, I. (1990). Estrategias de aprendizaje. En C. A. Coll, A. Marchesi, y J. Palacios (Comps.), Desarrollo psicológico y educación. Vol. Il Psicología de la Educación, (pp. 199-222). Madrid, España: Alianza

Sandía-Rondel, L. (2004). Metacognición en niños: una posibilidad a partir de la teoría de Vygotskiana. Acción Pedagógica, 13(2), 128-135. Recuperado de https://dialnet.unirioja.es/servlet/articulo?codigo $=2970390$

Schraw, G. \& Dennison, R. S. (1994). Assesing Metacognitive Awareness. Contemporary Educational Psychology, 19, 460-475.

Schraw, G., y Moshman, D. (1995). Metacognitive Theories. Educational Psychology Review 7(4), 351-371. Recuperado de https://digitalcommons.unl.edu/cgi/viewcontent.cgi?article=1040\&context=edpsychpap ers

Torres-Salas, M. (2010). La enseñanza tradicional de las ciencias versus las nuevas tendencias educativas. Revista Electrónica Educare, 14(1), 131-142. Recuperado de http://www.revistas.una.ac.cr/index.php/EDUCARE/article/view/1515

Análisis de las propiedades psicométricas del inventario Reflexiones sobre el estudio universitario

Racín Ciselle Fernández-Da Lama

DII: http://dx.dai.org//0.22458/caes.vlliz.3318

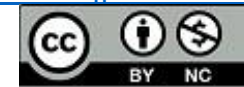

Artícula prategida por licencia Creative Cammans 
UNESCO. (1998). Declaración mundial sobre la educación superior en el siglo XXI: una visión $y$ acción. Recuperado de http://www.unesco.org/education/educprog/wche/declaration_spa.htm

Vrugt, A. y Oort, F. (2008). Metacognition, achievement goals, study strategies and academic achievement: Pathways to achievement. Metacognition and Learning, 3(2), 123-146. doi.org/10.1007/s11409-008-9022-4

Young, A. y Fry, J. (2008). Metacognitive awareness and academic achievement in college students. Journal of Scholarship of Teaching and Learning, 8(2), 1-10. Recuperado de https://scholarworks.iu.edu/journals/index.php/josot//article/view/1696 